\section{(C) OPEN ACCESS}

\title{
Shorter pulse width reduces gait disturbances following deep brain stimulation for essential tremor
}

\author{
Daniel Kroneberg, ${ }^{\oplus 1}$ Siobhan Ewert, ${ }^{1}$ Anne-Christiane Meyer, ${ }^{1}$ Andrea A Kühn ${ }^{1,2}$
}

- Additional material is published online only. To view please visit the journal online (http://dx.doi.org/10.1136/ jnnp-2018-319427).

${ }^{1}$ Charité - Universitätsmedizin Berlin, Department of Neurology, Movement Disorder and Neuromodulation Unit, Berlin, Germany

${ }^{2}$ Charité - Universitätsmedizin Berlin, NeuroCure Cluster of Excellence, Berlin, Germany

\section{Correspondence to} Professor Andrea A Kühn, Department of Neurology, Movement Disorder and Neuromodulation Unit, Charité Universitätsmedizin Berlin, Berlin 10117, Germany; andrea. kuehn@charite.de

Received 10 August 2018 Revised 12 December 2018 Accepted 4 January 2019 Published Online First 14 February 2019

\begin{abstract}
Objective Gait disturbances are frequent side effects occurring during chronic thalamic deep brain stimulation (DBS) in patients with essential tremor (ET). Adapting stimulation settings to shorter pulse widths has been shown to reduce side effects of subthalamic DBS. Here, we assess how a reduction of pulse width changes gait performance of affected patients.

Methods Sensor-based gait assessment was performed to record spatiotemporal gait parameters in 10 healthy subjects (HS) and 7 patients with ET with gait disturbances following thalamic DBS. Patients were tested during standard DBS, after 72 hours of stimulation withdrawal and at least 30 days after adjusting DBS settings to a shorter pulse width of $40 \mu \mathrm{s}$ (DBS40PW).

Results Patients with ET on standard DBS showed significantly higher variability of several spatiotemporal gait parameters compared with HS. Variability of stride length and range of motion of the shanks significantly decreased OFF DBS as compared with standard DBS. This improvement was maintained over 30 days with DBS40PW while providing effective tremor suppression in six out of seven patients.
\end{abstract}

Conclusion Shorter pulse widths may reduce gait disturbances in patients with ET that are induced by DBS while preserving a level of tremor suppression equal to standard stimulation settings.

\section{INTRODUCTION}

Thalamic deep brain stimulation (DBS) is a highly effective therapy for patients with otherwise therapy-refractory essential tremor (ET). ${ }^{1}$ Frequent side effects of thalamic DBS include dysaesthesia, speech disturbances and dysphagia. ${ }^{2}$ Gait disturbances may also occur with a delay of several months after activation of DBS and have been discussed as a stimulation-induced network dysfunction due to antidromic cerebellar activation. ${ }^{3}$ A subsequent increase of immobility and risk of falling may decrease the quality of life in these patients substantially. ${ }^{4}$ Studies on the prevalence of stimulation-associated gait disturbances report up to $50 \%$ of patients experiencing worsening of gait following thalamic DBS. Age, disease severity and preoperative gait difficulties have been identified as risk factors. $^{24}$

As DBS-naïve patients with advanced ET may exhibit balance impairment ${ }^{56}$ and increased spatiotemporal variability of lower limb movements similar to those seen in cerebellar ataxia, ${ }^{7}$ the differentiation between progression of disease-related gait disturbances and DBS-induced side effects ${ }^{8}$ remains challenging.

Antidromic activation of the cerebellar nodule via the uncinate tract from the subthalamic area has been discussed as a possible mechanism inducing gait ataxia in patients with ET with thalamic DBS. ${ }^{3}$ Previous studies had identified distinct electrophysiological properties of fibre tracts mediating tremor suppression and limb ataxia. ${ }^{9-11}$ Based on differences in axon diameters and chronaxies, shortening pulse widths would allow a more selective activation of cerebellothalamic fibres, which mediate tremor control without induction of ataxia.

Shorter pulse widths $(<60 \mu \mathrm{s})$ have been demonstrated to reduce side effects while maintaining clinical efficacy in patients with Parkinson's disease and DBS of the subthalamic nucleus. ${ }^{12} 13$ Shortterm beneficial clinical effects of thalamic DBS at short pulse widths were shown in a small cohort of patients with $\mathrm{ET},{ }^{14}$ and objective measures of change in gait parameters have been obtained a single case report. ${ }^{15}$

In this study, we used instrumented gait assessment technology to assess the reversibility of gait disturbances after pausing DBS and to quantify the beneficial effect of adjusting pulse widths to $40 \mu \mathrm{s}$, exploring the therapeutic potential of shorter pulse widths on stimulation-associated gait disturbances.

\section{PATIENTS AND METHODS \\ Study population}

Seven patients (four female, age 70.6 \pm 7.4 , $28.6 \pm 13.8$ years' disease duration) with deterioration of gait following DBS for ET (average time of chronic stimulation: $56.4 \pm 31.9$ months; clinical details in table 1) were included in the study. Only two of the seven patients had mentioned occasional falls ( $\leq 1$ per month) as a result of the decreased gait stability within the last 6 months prior to the study. All patients had Activa PC pulse generators with 3387 electrodes.

Each participant had previously undergone multiple $(>5)$ thorough attempts to improve gait difficulties by adjustment of stimulation parameters, including multiple monopolar reviews, change of active contacts including bipolar and interleaving stimulation settings and stimulation frequencies. The final setting for best tremor suppression found in this process was defined as ON (table 1). Importantly, all patients showed satisfactory tremor control with initial monopolar settings during the first year of DBS (tremor suppression of $\geq 50 \%$, range 50\%-95\%). 
Table 1 Clinical details

\begin{tabular}{|c|c|c|c|c|c|c|c|c|c|c|c|}
\hline \multirow[b]{2}{*}{ Patient } & \multirow[b]{2}{*}{ Sex } & \multirow[b]{2}{*}{ Age } & \multirow{2}{*}{$\begin{array}{l}\text { Disease } \\
\text { duration } \\
\text { (years) }\end{array}$} & \multirow{2}{*}{$\begin{array}{l}\text { Months since } \\
\text { DBS surgery }\end{array}$} & \multicolumn{5}{|c|}{ Stimulation parameter ON DBS } & \multirow{2}{*}{$\begin{array}{l}\text { Stimulation } \\
\text { amplitude } \\
\text { at DBS40PW } \\
\text { V }\end{array}$} & \multirow[b]{2}{*}{ Tremor medication } \\
\hline & & & & & & Contacts & $\mathrm{Hz}$ & V & $\mu s$ & & \\
\hline \multirow[t]{2}{*}{ ET1 } & $\mathrm{F}$ & 72 & $>50$ & 15 & $\mathrm{R}$ & $0-, 1-, 2+$ & 130 & 2.0 & 60 & 1.9 & Metoprolol 100 mg 1-0-1 \\
\hline & & & & & $\mathrm{L}$ & $1-{ }^{*} / 2{ }^{*}$ & 130 & $1.4 / 2.0$ & 60 & $1.4 / 2.0$ & \\
\hline \multirow[t]{2}{*}{ ET2 } & $\mathrm{F}$ & 72 & 38 & 19 & $\mathrm{R}$ & $0-* / 1-*$ & 130 & $0.9 / 0.8$ & 60 & $0.9 / 0.8$ & Propranolol 80 mg 1-0-1 \\
\hline & & & & & $\mathrm{L}$ & $0-{ }^{*} / 1-*$ & 130 & $1.2 / 2.7$ & 60 & $1.3 / 2.8$ & \\
\hline \multirow[t]{2}{*}{ ET3 } & M & 72 & 17 & 69 & $\mathrm{R}$ & $0-, 1-, 2+$ & 125 & 1.9 & 60 & 1.8 & None \\
\hline & & & & & L & $0-/ 1-$ & 125 & 1.6 & 60 & 1.1 & \\
\hline \multirow[t]{2}{*}{ ET4 } & M & 76 & 16 & 60 & $\mathrm{R}$ & $1-^{*} / 2-^{*}$ & 130 & $1.5 / 1.2$ & 60 & $1.6 / 1.6$ & None \\
\hline & & & & & L & $0-^{*} / 1-^{*}$ & 130 & $1.7 / 1.5$ & 60 & $2.5 / 2.5$ & \\
\hline \multirow[t]{2}{*}{ ET5 } & $\mathrm{F}$ & 73 & 20 & 117 & $\mathrm{R}$ & $2-{ }^{*} / 3-*$ & 110 & $1.6 / 1.6$ & 60 & $2.4 / 2.4$ & Primidone 250 mg 0-0-1 \\
\hline & & & & & $\mathrm{L}$ & $0-13-$ & 110 & 2.4 & 60 & 3.4 & Metoprolol $47.5 \mathrm{mg} 1-1-0$ \\
\hline \multirow[t]{2}{*}{ ET6 } & M & 54 & 40 & 67 & $\mathrm{R}$ & $2-3-$ & 130 & 2.3 & 60 & 2.5 & None \\
\hline & & & & & $\mathrm{L}$ & $0-1-$ & 130 & 2.0 & 60 & 2.1 & \\
\hline \multirow[t]{2}{*}{ ET7 } & $\mathrm{F}$ & 75 & 19 & 48 & $\mathrm{R}$ & $1-$ & 160 & 2.5 & 60 & 2.9 & Propranolol 40 mg 1-1-1 \\
\hline & & & & & $L$ & $1-$ & 160 & 3.7 & 60 & 4.1 & Primidone $250 \mathrm{mg}$ 0-0-1 \\
\hline Mean & & $70.6 \pm 7.4$ & $28.6 \pm 13.8$ & $\begin{array}{l}56.4 \pm 31.9 \\
\text { months DBS }\end{array}$ & & & & & & & \\
\hline
\end{tabular}

Clinical details of patient cohort.

*Interleaved stimulation.

DBS, deep brain stimulation; DBS40PW, parameter setting after reduction of pulse width to $40 \mu \mathrm{s}$; ET, essential tremor; F, female; L, left hemisphere; M, male; ON DBS, initial stimulation parameter setting; $R$, right hemisphere.

DBS electrodes were localised with Lead-DBS (V.2.1.0) toolbox ${ }^{16}$ using preoperative MRI and postoperative CT or MRI. All electrodes were located in the subthalamic area and ventral thalamus, slightly ventrally to the target published by Papavassiliou et l $^{17}$ (figure 1A,B).

Ten healthy subjects (five female, age $74.8 \pm 3.2$ ) with no subjective gait difficulties, no neurological disorders or any history of conditions affecting gait were recruited for comparison.

\section{Study protocol}

Access codes provided by Medtronic allowed programming of pulse widths shorter than $60 \mu \mathrm{s}$. A pulse duration of $40 \mu \mathrm{s}$ was chosen as it is closest to the chronaxy of the cerebellothalamic fibres. ${ }^{9}$ Clinical and gait parameters were assessed at three different stimulation conditions: (1) ON DBS (at standard parameters), (2) OFF DBS (after 3 days of unilateral withdrawal of stimulation corresponding to the non-dominant hand followed by one night of bilateral withdrawal of stimulation), and (3) at parameter setting after reduction of pulse width to $40 \mu \mathrm{s}$ (DBS40PW) (after at least 30 days [mean: 66 days; range 31-97] of chronic stimulation at $40 \mu$ s pulse width). If necessary, amplitudes were increased until the previous degree of tremor suppression was restored, whereas active contact(s) and stimulation frequency were left unchanged (table 1).

\section{Clinical assessment}

Axial items (1-3) of the Scale for Assessment and Rating of Ataxia (SARA) were used to assess the severity of ataxia in gait and trunk. Tremor severity was rated using the Fahn-Tolosa-Marin Tremor Rating Score items 1-9. Patients were asked to self-evaluate changes of gait performance on a Global Impression of Subjective Change Scale (GIC-S) (see figure 1).

\section{Quantitative gait assessment}

Kinematic gait analysis was performed using body-worn sensors (Mobility Lab, APDM, V.1.0, Portland,USA) attached to the shanks, wrists, sternum and lower waist. Patients walked $50 \mathrm{~m}$ at a comfortable gait speed. The Mobility Lab software ${ }^{18}$ was used to extract lower limb gait parameters from raw data. Coefficient of variation $(\mathrm{CoV})$, defined as the SD divided by the mean, was calculated for each gait parameter. We focused on stride length, stride velocity, stride time, cadence and range of motion (RoM) of the knees and shanks and their respective CoVs. These parameters have previously been reported to be altered in cerebellar diseases ${ }^{19}$ and to be differentially modulated by therapeutic and supratherapeutic stimulation.?

\section{Statistical analysis}

Given the cohort size, non-parametric Friedman test was used to determine the effect of stimulation condition on gait parameters, SARA score, tremor and GIC. Post-hoc comparisons were calculated using Wilcoxon signed-rank test. For comparison with healthy subjects, Mann-Whitney U tests were used. P values were adjusted for multiple comparisons using false discovery rate.

\section{RESULTS}

Group averages of all gait parameters from healthy controls and patients in all stimulation conditions are reported in an online supplementary table.

At baseline (DBS ON), patients with ET showed significantly higher values for $\mathrm{CoV}$ of stride length $(p=0.003)$, RoM of the shanks $(p=0.003)$ and RoM of the knees $(p=0.0001)$ compared with healthy subjects. Modulation of DBS parameters led to changes in behavioural performance, with increased tremor and reduced ataxia scores in the OFF DBS condition. Importantly, DBS40PW also reduced ataxia but maintained tremor suppression. Consistently, Friedman tests revealed a significant effect of 'Stimulation condition' on gait parameters $\mathrm{CoV}$ of stride length $(p=0.005)$ and CoV of the shank RoM $(p=0.018)$. Post-hoc tests showed a significant reduction of CoV stride length OFF DBS $(\mathrm{p}=0.016)$ and at DBS40PW $(\mathrm{p}=0.016)$, as well as a significant 

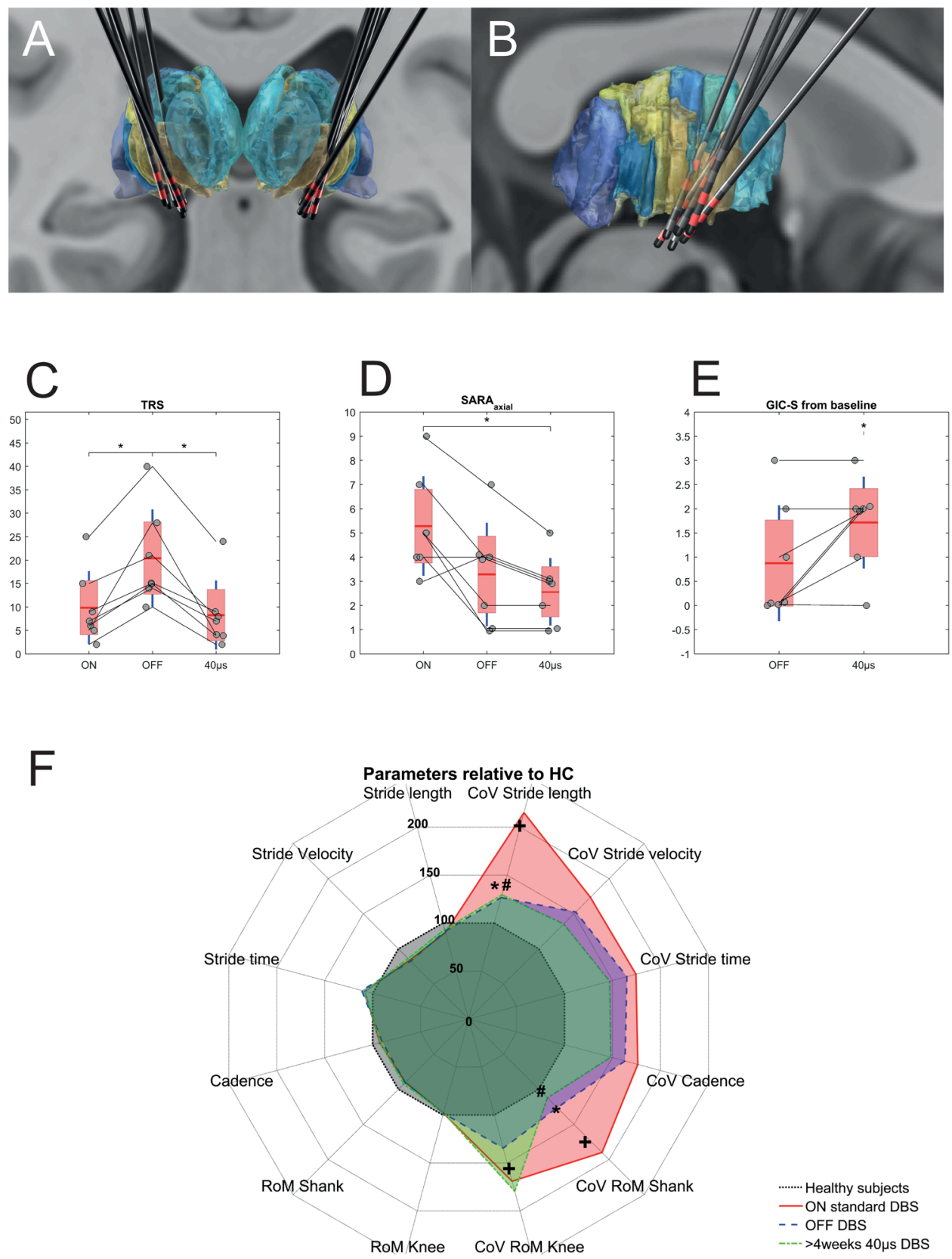

Figure 1 (A) Coronal view of reconstructed electrodes in MNI space in relation to the thalamus (DISTAL Atlas, Ewert 2017)22. The ventral intermediate nucleus is coloured light brown. Active contacts for each patient are highlighted in red colour. The average $x, y$ and $z$ coordinates in the MNI space of the lowermost contact were $15.37( \pm 1.54),-11.37( \pm 1.84)$ and $2.80( \pm 1.91)$ for the right electrode and $-15.94( \pm 1.06),-10.75( \pm 1.91)$ and $2.57( \pm 2.67)$ for the left electrode, respectively. (B) Sagittal view of electrode reconstructions of the right hemisphere. (C-E) Clinical outcome under the three test conditions: ON DBS, OFF DBS and after >30 days of adjusted DBS with $40 \mu$ s pulse (DBS4OPW). (C) TRS: Fahn-Tolosa-Marin Tremor Rating Score (items 1-9). (D) SARA $_{\text {axial }}$ subscore. (E) Global Impression of Subjective Change (GIC-S) OFF DBS and after $>30$ days of adjusted DBS4OPW compared with DBS ON (1=very much improved; $2=$ =much improved; $3=$ =minimally improved; $4=$ unchanged; $5=$ =minimally worse; $6=$ much worse; $7=$ =very much worse). Values of individuals are displayed as circles over the group mean (red line), SEM (red area) and SD (blue line). (F) Spatiotemporal gait parameters and their coefficients of variation (CoVs) relative to measures obtained in healthy controls ( $\mathrm{HC}$, black dotted line=baseline of $100 \%$ ). Right half of the diagram shows CoVs of parameters, left half the average parameter relative to HC. Three stimulation conditions are depicted: ON standard DBS (red line), OFF DBS after stimulation withdrawal (blue line) and after $>30$ days with $40 \mu \mathrm{SBBS}$ (DBS40PW, green line). + Indicates significant differences of ET_ON gait parameters compared with HC. * Indicates significant change of gait parameters OFF stimulation compared with standard DBS. \# Indicates significant change of gait parameters with $40 \mu \mathrm{S}$ PW compared with standard DBS. Note the significant reduction in CoV OFF DBS that is maintained with DBS4OPW. All p values corrected using false discovery rate. Abbreviations: DBS - deep brain stimulation; MNI - Montreal Neurological Institute; RoM- range of motion; SARA - Scale for Assessment and Rating of Ataxia. 
reduction of CoV shank RoM OFF DBS $(p=0.03)$ and at DBS40PW $(p=0.016)$ compared with ON DBS (figure 1E).

Tremor scores increased with stimulation OFF $(19.3 \pm 11.5)$ compared with ON $(9.9 \pm 7.8 ; \mathrm{p}=0.02)$ and DBS40PW $(8.3 \pm 7.4$; $\mathrm{p}=0.02$; figure 1C). SARA axial items (1-3) decreased with stimulation OFF $(3.3 \pm 2.1 ; \mathrm{p}=0.06)$ and DBS40PW $(2.6 \pm 1.4$; $\mathrm{p}=0.03)$ compared with $\mathrm{ON}(5.3 \pm 2.1$; see figure $1 \mathrm{D})$.

Global impression of change revealed a significant difference for ON DBS compared with DBS40PW $(2.3 \pm 1.0 ; \mathrm{p}=0.03)$ but not OFF DBS $(3.1 \pm 1.2 ; \mathrm{p}=0.25$; figure $1 \mathrm{E})$.

None of the seven patients experienced falls during the gait assessment or test period in either of the three stimulation conditions.

\section{DISCUSSION}

We showed that stimulation-induced gait disturbances are reversible when pausing thalamic DBS and that this improvement can be maintained when DBS parameters are changed to shorter pulse widths while DBS remained efficient for tremor suppression in our patients.

This clinical observation is corroborated by objective measurements of spatiotemporal gait parameters that showed a reduction in the CoV OFF DBS and with DBS40PW as compared with standard DBS for stride length and the shank RoM. These parameters were also increased during DBS ON compared with healthy controls, indicating a change towards normalisation with DBS40PW (figure 1F, online supplementary video).

Withdrawing stimulation for several days ${ }^{3}$ to differentiate stimulation-induced gait disturbances from disease progression offers no therapeutical benefit, as tremor inevitably re-emerges and gait disturbances return once stimulation is reactivated with unchanged stimulation parameters.

Sensor-based quantitative gait assessment revealed a greater variability of spatial and temporal gait parameters in patients with ET ON DBS compared with healthy subjects.

Similar results using the same sensor system have been reported in patients with Spinocerebellar Ataxia Type 14 (SCA14), which is considered a pure cerebellar ataxia, thus supporting the hypothesis of cerebellar involvement leading to gait alterations in ET ON DBS.

The CoV of stride length decreased after withdrawal of DBS, and this change towards normalisation in gait parameters was maintained over several weeks of DBS at shorter pulse width. Importantly, while switching off DBS resulted in reoccurrence of tremor, DBS40PW provided efficient tremor control in all patients.

ET is considered a network disorder involving the cerebellum, motor cortex and thalamus. ${ }^{620}{ }^{21}$ DBS is believed to mediate its effect through modulation of abnormal signalling in cortico-thalamo-cortical and cerebello-thalamo-cortical pathways $^{7}$ via the dentate-thalamic tract. ${ }^{9}$ Inadvertent activation of cerebello-rubro-spinal fibres in anatomical vicinity may explain disruption of goal-directed movements, leading to ataxia in the upper ${ }^{9}$ and lower ${ }^{7}$ extremities.

Our results support existing evidence that these fibre tracts may be selectively activated using different pulse widths for DBS due to their distinct electrophysiological properties. ${ }^{9}{ }^{12-14}$ Although changes in stimulation amplitudes at DBS40PW to baseline were inconsistent, previous attempts of parameter optimisation by voltage reduction had led to reduced tremor control, while shorter pulse widths provided efficient tremor control and reduced stimulation-induced side effects in our patients. However, applicability of this finding is currently limited as not all commercial neurostimulators support pulse widths $<60 \mu$ s. During battery replacement, however, it can be considered to use adapter kits to connect pulse generators and electrodes from different manufacturers. ${ }^{15}$ A firmware change with limited availability was required for our subjects, explaining the small number of patients.

Our results add new quantitative evidence for clinical efficacy of shorter pulse widths to address stimulation-induced side effects and optimise outcome in patients with thalamic stimulation. ${ }^{1011} 15$ Nevertheless, our study bears limitations: the SARA score is a rating scale for ataxia not validated for patients with ET. Due to sample size, unblinded assessment and short follow-up period, our study should be considered preliminary and future trials should verify the long-term clinical impact of short pulse widths for thalamic DBS, especially if used as an initial parameter setting.

Contributors DK and AK: study concept and design, project execution, data acquisition and processing, statistical analysis, manuscript preparation. SE: project execution, data processing, manuscript critique. A-CM: project execution, data acquisition, statistical analysis, manuscript critique.

Funding Grant from the German Research Council (DFG) Klinische Forschergruppe KFO247 and grant from NeuroCure, Centre of Excellence, Charité, Berlin to AK.

Competing interests DK, SE and A-CM declare no competing interests or financial dependencies. AK received honoraria from Medtronic, Boston Scientific and St Jude Medical, is member of an advisory board for Boston Scientific, received a travel grant from Boston Scientific, and received a research grant from Medtronic.

\section{Patient consent for publication Not required.}

Ethics approval The study was approved by the institutional review board of the Charité.

Provenance and peer review Not commissioned; externally peer reviewed.

Open access This is an open access article distributed in accordance with the Creative Commons Attribution Non Commercial (CC BY-NC 4.0) license, which permits others to distribute, remix, adapt, build upon this work non-commercially, and license their derivative works on different terms, provided the original work is properly cited, appropriate credit is given, any changes made indicated, and the use is non-commercial. See: http://creativecommons.org/licenses/by-nc/4.0

\section{REFERENCES}

1 Schuurman PR, Bosch DA, Bossuyt PM, et al. A comparison of continuous thalamic stimulation and thalamotomy for suppression of severe tremor. N Eng/ J Med 2000;342:461-8

2 Pahwa R, Lyons KE, Wilkinson SB, et al. Long-term evaluation of deep brain stimulation of the thalamus. J Neurosurg 2006;104:506-12.

3 Reich MM, Brumberg J, Pozzi NG, et al. Progressive gait ataxia following deep brain stimulation for essential tremor: adverse effect or lack of efficacy? Brain 2016;139:2948-56

4 Hwynn N, Hass CJ, Zeilman P, et al. Steady or not following thalamic deep brain stimulation for essential tremor. J Neurol 2011;258:1643-8.

5 Kronenbuerger M, Konczak J, Ziegler W, et al. Balance and motor speech impairment in essential tremor. Cerebellum 2009;8:389-98.

6 Stolze $\mathrm{H}$, Petersen $\mathrm{G}$, Raethjen J, et al. The gait disorder of advanced essential tremor. Brain 2001;124:2278-86.

7 Fasano A, Herzog J, Raethjen J, et al. Gait ataxia in essential tremor is differentially modulated by thalamic stimulation. Brain 2010;133:3635-48.

8 Favilla CG, Ullman D, Wagle Shukla A, et al. Worsening essential tremor following deep brain stimulation: disease progression versus tolerance. Brain 2012;135:1455-62.

9 Groppa S, Herzog J, Falk D, et al. Physiological and anatomical decomposition of subthalamic neurostimulation effects in essential tremor. Brain 2014;137:109-21.

10 Moldovan AS, Hartmann CJ, Trenado C, et al. Less is more - Pulse width dependent therapeutic window in deep brain stimulation for essential tremor. Brain Stimul 2018;11:1132-9.

11 Choe CU, Hidding U, Schaper M, et al. Thalamic short pulse stimulation diminishes adverse effects in essential tremor patients. Neurology 2018;91:e704-13.

12 Steigerwald F, Timmermann L, Kühn A, et al. Pulse duration settings in subthalamic stimulation for Parkinson's disease. Mov Disord 2018;33:165-9.

13 Reich MM, Steigerwald F, Sawalhe AD, et al. Short pulse width widens the therapeutic window of subthalamic neurostimulation. Ann Clin Trans/ Neurol 2015;2:427-32. 
14 Reich MM, Pozzi NG, Brumberg J, et al. Reply: clinical approach to delayed-onset cerebellar impairment following deep brain stimulation for tremor. Brain 2017;140.

15 Soh D, Lozano AM, Fasano A. Hybrid deep brain stimulation system to manage stimulation-induced side effects in essential tremor patients. Parkinsonism Relat Disord 2018.

16 Horn A, Kühn AA. Lead-DBS: a toolbox for deep brain stimulation electrode localizations and visualizations. Neuroimage 2015;107:127-35.

17 Papavassiliou E, Rau G, Heath S, et al. Thalamic deep brain stimulation for essential tremor: relation of lead location to outcome. Neurosurgery 2008;62(Suppl 2):884-94.

18 Mancini M, King L, Salarian A, et al. Mobility lab to assess balance and gait with synchronized Body-worn sensors. J Bioeng Biomed Sci 2011;1:007.
19 Ilg W, Golla $\mathrm{H}$, Thier $\mathrm{P}$, et al. Specific influences of cerebellar dysfunctions on gait. Brain 2007;130:786-98.

20 Louis ED, Faust PL, Vonsattel JP, et al. Neuropathological changes in essential tremor: 33 cases compared with 21 controls. Brain 2007;130:3297-307.

21 Schnitzler A, Münks C, Butz M, et al. Synchronized brain network associated with essential tremor as revealed by magnetoencephalography. Mov Disord 2009:24:1629-35.

22 Ewert S, Plettig P, Li N, et al. Toward defining deep brain stimulation targets in MNI space: a subcortical atlas based on multimodal MRI, histology and structural connectivity. Neuroimage 2018;170:271-82. 